\title{
LA CUEVA DEL TESORO - RINCON DE LA VICTORIA (Málaga, España)
}

\author{
Antonio Guzmán, Fernando Mendoza, José Luís Gutiérrez*
}

\begin{abstract}
The Treasure Cave, Rincón de la Victoria (Málaga, España) ]
«La Cueva del Tesoro» (The Treasure Cave) is located on the East, 10 kilometres from the city of Málaga, in a coastal promontory called «El Cantal» (a small steep), which is situated in the municipality of Rincón de la Victoria.

The composition of land in this zone «El Cantal» is made up of limestone and there are many caves which are located within a radius of 2 kilometres, although some of them have already disappeared: la Cueva del Tesoro, la cueva del Hoyo de la Mina, Cuevas de Navarro, Cuevas de la Cantera, Cueva de los Molinos, Cueva de la Raja del Humo, etc. The best known of these caves and the only one who can be visited is the Cueva del Tesoro. It has been also known by some other names, such as the Cueva del Higuerón or the Cueva del Suizo.

The Cueva del Tesoro has its origin in the sea coast depths; that is why its morphology is made up of halls, gorges and columns. Then, and because of the upheaval of «E1 Cantal» the cave emerged out of the sea. Finally, fresh water percolation resulted in the formation of some stalactites and stalagmites, although they are of less importance within the whole cave.

This cave, which was already used as shelter for 8 months by Marcus Crassus (according to a legend) in 86 B.C. when he was prosecuted, it was also used, according to another tradition, to hide the Almoravid treasure in the 12th century.

This treasure has been searched for by some people in the last two hundred years. One of them was. the Swiss, Antonio de la Nari, who died because of an explosion inside the cave in 1847. Professor Manuel Laza Palacios from Málaga, owner of the cave, has been the best specialist and treasure searcher. He was an exceptional person, has knew how to keep alive hopefully the old legend throughout his whole life.

Besides, the cave has provided some important archaelogical discoveries: Father Breuil found out some cave paintings in 1918 and excavations have been recently carried out by Mr. Manuel Laza. A rather interesting material appeared, such as Neolithic pottery, lithic industry (the most important object is an Upper Paleolithic arrow-head), human and animal remains. According to these discoveries, the presence of human beings in this cave is proved since the Paleolithic.

There are also a series of legends and traditions related to the Cueva del Tesoro. So, in this frame, it has been given for sure and for a very long time that the ghost of the Swiss still appears in «El Cantal» to look for the treasure. Another tradition, studied by Mr. Laza, places the sanctuary of the old goddess Noctiluce inside a hall of the cave.
\end{abstract}

\section{RIASSUNTO [La grotta del Tesoro, Rincón de la Victoria (Málaga , España)]}

«La Cueva del Tesoro» (Grotta del Tesoro) si apre a $10 \mathrm{~km}$ ad Est della città di Málaga, in un promontorio denominato «El Cantal» (piccolo scoscendimento) nel territorio del comune di Rincón de la Victoria.

Il terreno nella zona del «El Cantal» consiste in calcare e vi sono numerose cavità nel

* c/o Cueva del Tesoro, Ayuntamiento de Rincón de la Victoria, E-29730 MALAGA (España) 
raggio di 2 chilometri, anche se di alcune di esse si è persa traccia: la Cueva del Tesoro, la cueva del Hoyo de la Mina, Cuevas de Navarro, Cuevas de la Cantera, Cueva de los Molinos, Cueva de la Raja del Humo, ecc. La più nota di queste grotte e l'unica che può essere visitata è la Cueva del Tesoro. Essa è anche nota sotto altri nomi come la Cueva del Higuerón o la Cueva del Suizo.

La Cueva del Tesoro ha la sua origine nell'interno della costa marina e ciò spiega la presenza di sale, gole e colonnati. Successivamente la grotta è emersa dal mare per l'innalzamento del «El Cantal». Infine, la percolazione di acque dolci ha portato alla formazione di alcune stalattiti e stalagmiti che, tuttavia, non hanno eccessiva rilevanza in tutto l'insieme della grotta.

Questa cavita, che era già stata il rifugio per 8 mesi di Marco Crasso (secondo una leggenda) nel 86 a.C. quando era sotto processo, pare sia stata adoperata, secondo un'altra tradizione, per nascondere il tesoro degli Almoravidi nel $12^{\circ}$ secolo.

Questo tesoro è stato oggetto di ricerche da parte di varie persone negli ultimi duecento anni. Una di queste era lo svizzero Antonio de la Nari, che morì all'interno della grotta a causa di un'esplozione nel 1847. Il professor Manuel Laza Palacios from Málaga, proprietario della grotta, è stato il miglior specialista e cercatore di tesori. Era una persona eccezionale che ha saputo tener viva la vecchia leggenda nel corso di tutta la sua vita.

Inoltre, la grotta è stato il luogo di importanti scoperte preistoriche: l'Abate Breuil trovo alcune pitture rupestri nel 1918 e Manuel Laza ha effettuato recentemente degli scavi. E' stato così rinvenuto del materiale piuttosto interessante, come della ceramica neolitica, dell'industria litica (l'oggetto più importante à uns punta di freccia del Paleolitico superiore) e dei resti umani e di animali. Secondo queste scoperte la presenza umana è documentata fin dal Paleolitico.

Vi è anche tutta una serie di leggende e di tradizioni legate alla Cueva del Tesoro. Così, a questo proposito, a lungo si è dato per certo che lo spettrop dello svizzero appare ancora nella zona del «El Cantal» alla ricerca del tesoro.

Un'altra tradizione, studiata dal Laza, pone in una sala della grotta il santuario dell'antica dea Noctiluca.

\section{SITUACION}

A unos 10 kilómetros al este de la ciudad de Málaga se encuentra la Cueva del Tesoro, en el municipio costero de Rincón de la Victoria. La Cueva se halla sobre uno de los Cantales (o pequeños acantilados), que en forma de promontorios calizos de la época jurásica se a soman al mar por esta parte de la costa mediterránea. El mar es el principal agente erosivo que ha contribuito a la formación del actual relieve de los Cantales. A través de milenios se han ido originando las cuevas y acantilados que hoy son lo característico de este paisaje.

\section{ORIGEN GEOLOGICO DEL CANTAL}

a) El Cantal se originó en una cuenca de sedimentación submarina donde habia plegados y depositados materiales silíceos (filitas). Sobre estos terrenos se depositaron varias capas de material calizo que se fueron sedimentando sobre la pizarra.

b) Más tarde la caliza se fue plegando junto con los materiales silíceos y, juntos, comenzaron su movimiento de ascensión creado por los cambios 
isostáticos. Al salir fuera del mar, los materiales calizos se fueron erosionando hasta desgastarse y aflorar a la superficie los meteriales silíceos situados debajo.

c) Con posterioridad, y gracias de nuevo a los movimientos isostáticos, emergieron del fondo del mar los terrenos calizos que se encontraban en los extremos de la plataforma de sedimentación y que no habían sufrido la erosión, ya que habían quedado debajo del agua. Estos dos extremos son las escamas (restos de anteriores composiciones geológicas) calizas de El Cantal y de Comares. Ambas son el último reducto de los terrenos calizos en esta zona.

d) La plataforma de abrasión, o rompeolas de El Cantal actual se encuentra bajo un acantilado calizo que emergió del mar gracias a los movimiento isostáticos a los que se ve sometida la costa. Al repertirse varias veces este movimiento, podemos observar la existencia de antiguos acantilados y plataformas.

\section{CARACTERISTICAS MORFOLOGICAS DE LA CUEVA DEL TESORO.}

El complejo subterráneos de El Cantal está formado por varias cavidades que poseen vestigios culturales desde el Musteriense así como arte rupestre. Este complejo está formado por dos cavidades principales:

a) La cueva del Tesoro, llamada anteriormente del Suizo y más antiguamente del Higuerón. Tiene aproximadamente unos 500 metros de galerias.

b) La Cueva de la Victoria, en estado actual de abandono y degradación.

Centrándonos en la Cueva del Tesoro, hay que decir que tiene unas formaciones calizas típicas de las cuevas formadas en las profundidades marinas. La acción de las corrientes y el oleaje hace que se vayan erosionando, las partes más blandas y queden en forma de las columnas vetas de caliza más fuertes.

Más tarde, cuando las tierras emergieron, las filtraciones de agua dulce, han creado algunas estalactitas y estalagmitas. Sin embargo esto es los menos significativo de la cueva.

\section{LA CUEVA DEL TESORO DURANTE LA HISTORIA}

La Cueva del Tesoro ha estado vinculada a múltiples leyendas y hechos históricos relacionados con el supuesto tesoro que se cree que existe todavía escondido en su interior. Don Manuel Laza Palacio, profesor puy querido en Málaga y propietario de la cueva hasta su muerte acaecida en 1988, ha sido un entusiasta estudioso de la cueva y buscador del tesoro. Don Manuel era una persona muy atractiva que le supo buscar un sentido a su vida con la búsqueda de este tesoro durante cerca de 40 años. El interés de don Manuel no era materialista sino que lo que él pretendía era demostrar que varias 
intuiciones pueden llevar a un hombre a conseguir su objetivo. Don Manuel simplemente buscaba un tesoro aunque probablemente no lo iba a encontrar nunca.

Por los estudios de don Manuel Laza conocemos la historia de la cueva en las distintas épocas, Asimismo conservamos sus Cuadernos de Excavaciones donde se nos informa de los trabajos arqueológicos por él llevadas a cabo.

Los datos más antiguos que tenemos de la Cueva del Tesoro y de la leyenda de los Cinco Reyes se remonta al siglo XVIII. Un ilustrado local, don Cristobal Medina Conde, publica en 1789, bajo el seudónimo de Cecilio García de la Leña, la obra titulada Conversaciones Históricas Malagueñas. Según esta obra, fue utilizada la Cueva del Higuerón (actual Cueva del Tesoro) en el año 86 a. C. por Marco Craso para refugiarse durante 8 meses en los que fue perseguido por Mario y Cinna. La tradición del refugio de Craso en la cueva es más antigua porque el propio Medina Conde la recoge de un autor anterior, de finales del siglo XVII, que ya menciona también la del tesoro. Se trata de Fray Antonio Augustín de Milla y Suazo autor de una obra aún inédita titulada Historia Eclesiástica y Secular de Málaga y su Obispado.

\section{LA TRADICION DEL TESORO Y SU BUSQUEDA HASTA LA ACTUALIDAD.}

Es antigua la tradición oral y escrita que recoge la existencia de un tesoro escondido durante la época árabe en la Cueva del Higuerón. Esta tradición ya se refleja en la obra de Fray Antonio Agustín de Milla y Suazo (Siglo XVII).

Según don Manuel Laza, este tesoro fue mandado esconder por el emperador de los almorávides Tasufín ibn Alí en el siglo XII. Para ello se basa en algunos hallazgos y en el análisis de las fuentes de la época árabe en España.

El tesoro ha tenido diversos buscadores en los tres últimos siglos. Medina Conde nos relata una expedición a la cueva en ple no siglo XVII por 17 personas que, tras presenciar diversos hechos mágicos y maravillosos, salieron de la cueva con mucho miedo.

En la primera mitad del siglo XIX, un suizo, Antonio de la Nari, pasó varios años buscando el tesoro. A partir de entonces la cueva pasó a llamarse del Suizo. Este utilizó la pólvora para abrir nuevas galerías y fue el descubridor de una parte de la cueva que estaba taponada y oculta desde hacía siglos. El Suizo murió en 1847 víctima de una de sus explosiones provocadas en la cueva. Existe la tradición en la zona de que todavia se aparece como espectro para buscar el tesoro.

Don Manuel Laza ha sido el último buscador del tesoro durante 38 años. Hasta el último momento de su vida don Manuel creyó en la existencia del tesoro y los hallazgos que relaciona con el mismo son los siguientes:

a) Hallazgo en el siglo XVIII de una porción de granates (Según el relato de Medina Conde).

b) Aparición en las cercanías de un tesorillo de 6 monedas almorávides 
de oro de la época de Yusuf ibn Tasufín. Tanto este hallazgo como los siguientes son de la época de don Manuel Laza.

c) La rotura y desaparición de los niveles arqueologicos normales en todo gruta prehistórica en la parte del centro en una de las salas de la Cueva (Sala de la Virgen). Esta zona estaba constituida, cuando se excavó por tierra removida con mezcla de cerámicas neolíticas, objetos de sílex, huesos humanos, etc. El resto de la sala si conservaba intactos sus estratos normales.

d) Hallazgo de tres puertas o torcas que daban a la misma sala de la cueva y que habían sido taponadas de forma artificial con grandes piedras. Al quitar estas piedras aparecieron con ellas restos de cerámica árabe vidriada similar a la aparecida en la cercana ciudad árabe de Bezmiliana.

e) En la misma sala se encontró una sima llena de grandes piedras y barro. La posición de estos materiales hacía pensar que se trataba de un relleno artificial muy antiguo.

Estos hallazgos y otros posteriores hicieron creer a don Manuel Laza que se encontraba cada vez más cerca del tesoro y que siguiendo la investigación alguien lo encontrará alguna vez.

\section{LOS HALLAZGOS ARQUEOLOGICOS}

En 1918 visita la Cueva del Suizo el abate Breuil y nos deja una descripción de la misma en un articulo de la revista «L'Anthropologie», tomo XXXI, titulado «Nouvelles cavernes ornées paléolithiques dans la province de Málaga». Describe restos de pinturas rupestres que relaciona con las pinturas de signos rojos de la Cueva de la Pileta de Benaoján.

Los trabajos arqueológicos han sido desarrollados por don Manuel Laza y don Simeón Giménez Reyna en una zona muy pequeña de la cueva en la sala antes mencionada. Los suelos aparecieron revueltos y muy difíciles de adecuar a una estratigrafía. Se supone que todo está revuelto por antiguos buscadores del tesoro como el mítico suizo.

Los restos aparecidos en estas excavaciones, de los que se puede observar una muestra en el Museo Arqueológico Nacional, en Madrid, los clasificamos en los siguiendes grupos:

a) Cerámicas: Son numerosos vasos y fragmentos correspondientes al llamado Neolítico de las cuevas.

b) Industria lítica: Son numerosas las piezas de sílex aparecidas. Destacamos la punta de flecha clasificada por el profesor Luis Pericot como solutrense.

c) Restos humanos: Abundantes también. Destacamos dos cráneos completos.

d) Restos de animales: Hay gran abundancia de ellos. Destacamos un conchero y un hueso fósil de Bisón (Paleolítico Superior). 


\section{OTRAS LEYENDAS Y CURIOSIDADES}

En relación con la Cueva del Tesoro hay una serie de leyendas y curiosidades que forman parte de la tradición de estos pueblos marineros de la costa mediterránea. De entre ellas destacamos:

- La historia del suizo que dio nombre a la cueva durante años, Antonio de la Nari, posiblemente un licenciado de la guardia walona de los Reyes, al igual que la de su paisano Benedicto Mol, descrito por el inglés George Borrow (llamado don Jorgito en España) en su obra «La Biblia en España», se dedicó a la busca de tesoros. Atraido por la leyenda de los Cinco Reyes Moros, vino a Málaga y se dedicó a buscar èl tesoro de la Cueva del Higuerón. Varios escritores de la época dan fe de su trágica muerte, destrozado por la explosión de un barreno en 1847.

Una leyenda de terror y misterio se extendió por la zona en torno a la Cueva del Suizo (así llamada desde entonces). Durante la noche vagaba en pena su alma por las soledades del Cantal Alto. Muchos vecinos atestiguaban apariciones terroríficas.

- Según los estudios de don Manuel Laza, se sitúa en la cueva el antiguo Santuario de la Diosa Noctiluca. Festo Avieno en su poema latino «Ora Maritima», cita tres santuarios prehistóricos en el sur de España: uno consagrado a Hércules; otro dedicado a Venus en el Cabo de Gata en Almería; y el tercero en Cádiz a la diosa Noctiluca en Málaga. Esta diosa de la fecundidad, la vida y la muerte, era representada por los fenicios en forma de betilo (piedra basta) en la Tierra, y en los Cielos era la Luna en sus distintas fases. La monedas fenicias de Malaka (actual Málaga) representan el culto a esta divinidad bajo los mismos elementos.

En la cueva del Tesoro existe una formación pétrea (betilo) que nos recuerda las características de esta divinidad lunar de la moneda. Esto hace pensar, junto con la descripción del poema de Festo Avieno, que estamos ante el antiguo Santuario de la diosa Noctiluca.

\section{ESTADO ACTUAL DE LA CUEVA DEL TESORO}

La cueva del Tesoro se encuentra cerrada al público y detenidas su investigaciones desde hace cinco años.

Ultimamente el Ayuntamiento de Rincón de la Victoria, en cuyo término se encuentra la cueva, ha llegado a un acuerdo con los actuales propietarios para volver a abrirla y que se pueda disfrutar de sus bellezas por parte de los turistas que acuden a estas costas a pasar sus vacaciones, así como por todas las personas estudiosas o interesadas por estos temas.

Actualmente la cueva se encuentra en fase de estudio y reformas para poder abrirla en un futuro no muy lejano. 


\section{LAS CUEVAS CERCANAS - ESTADO DE CONSERVACION}

En el mismo Cantal y cerca de la Cueva del Tesoro se encuentra:

a) La cueva de la Victoria. Descubierta en 1939 y con un importante yacimiento arqueológico destruido desde hace tiempo. El Museo Arqueológico Provincial de Málaga conserva el material recogido.

Tambi0n se encontró en esta cueva una serie de pinturas esquemáticas de la 0poca neolítica. Su estado de conservación es bastante malo y en situación de abandono.

En otro Cantal situado entre 1 y 2 kilómetros, se encuentran una serie de cuevas muy deterioradas por la existencia de una fábrica de cemento que usa el Cantal como cantera de materia prima. Asimismo el impacto de la nueva autovía ha influido negativamente en la conservación de la cuevas. Entre ellas citamos:

b) Cueva del Hoyo de la Mina (o del Tío Leal), era conocida en 1833. En 1917 fue excavada por don Miguel Such, obteniendo materiales neolíticos y eneolíticos que se encuentran en el Museo Arqueológico de Málaga. Esta cueva ha desaparecido por el avance de la cantera de la fábrica.

c) Cuevas de Navarro. Cerca de la barriada de La Araña, donde se halla enclavada la fábrica de cemento, y en dirección a la Cala del Moral y Rincón de la Victoria, se encuentran una serie de cuevas (de la I a la VIII), llamadas de los Hermanos Navarro. Estas han ido desapareciendo por el avance de una cantera existente.

La más interesante es la llamada Navarro IV, formada por 1.156 metros de galerias en forma laberíntica. En su interior se encuentra un santuario solutrense con pinturas en rojo y ocre, descubierto en 1979.

d) Cuevas de la Cantera. Desde 1969 han ido apareciendo en los trabajos de la cantera de la fábrica de cemento las Cuevas de la Cantera (de la I a la III). Han ido desapareciendo asimismo con los trabajos de la propia cantera. La Cueva de la Cantera I, la más interesante, estaba formada por 200 metros de bellas galerías en $X$ con formaciones cristalinas. También apareció en ella abundante material prehistórico.

e) Cueva de los Molinos. Se encuentra también en la cantera de La Araña (fábrica de cemento) en dirección a Málaga. Consta de una galería de unos 40 metros donde han aparecido fragmentos de sílex paleolítico y restos de conchas.

f) Cueva de la Raja de Humo. Conocida anteriormente con los nombres de Montijano y del Higuerón. Pascual Madoz la cita con este nombre en su célebre «Diccionario Geográfico» en 1845. Se sitúa en el extremo de La Araña, a 200 metros de la Cala del Moral.

Sucevivas excavaciones han puesto de manifiesto un importante yacimiento paleolítico. Se conserva una gran cantidad lítico de la cuarcita perteneciente al Musteriense de técnica Levallois. También son abundantes los concheros de mejillones y lapas.

Su estado de conservación se ha visto recientemente afectado por la construcción de la nueva autovía. 


\section{BIBLIOGRAFIA}

Gimenez Reyna S., 1946 - Memoria Arqueológica de la Provincia de Málaga hasta 1946. Ministerio de Educación Nacional. Madrid.

Gimenez Reyna S. \& Laza Palacio M., 1964 - Informe de las Excavaciones en la Cueva del Higuerón o Suizo. Noticiario Arqueológico Hispánico, 6, Madrid.

Laza Palacio M., 1962 - La Cueva del Tesoro. Revista Gibralfaro, 11, Málaga.

Laza Palacio M., 1988 - El hombre que créa saber dónde había un tesoro. Primtel. Universidad de Málaga.

Lopez Moreno J.B., 1989 - Proyecto Interdisciplinar sobre la Cueva del Tesoro y su entorno. Instituto de Bachillerato, Rincón de la Victoria.

Perez Berrocal J.A. \& Moreno Wallace L., 1988 - Guía de la Cuevas de Málaga. Diputación Provincial de Málaga.

Perez de Barradas J., 1968 - Los primitivos pobladores de la Costa del Sol. Caja de Ahorros de Málaga. 